\title{
A new trend in sensitization to cockroach allergen: A cross-sectional study of indoor allergens and food allergens in the inland region of Southwest China
}

\author{
Wenting Luo, ${ }^{1 \dagger}$ Huixiong Chen, ${ }^{1,2 \dagger}$ Zehong Wu, ${ }^{1}$ Haisheng Hu, ${ }^{1}$ Wanbing Tang, ${ }^{1,2}$ Hao Chen, ${ }^{1}$ Baoqing Sun, ${ }^{1}$ Huimin Huang ${ }^{1}$
}

\begin{abstract}
Background: Despite the increasing prevalence of allergic disease, large-scale studies to investigate allergen sensitization have rarely been conducted in the inland region of Southwest China.

Objective: This study aimed to investigate the trend of allergen sensitization in mainland China from 2016 to 2017.

Methods: During the 2-year study period, from 2016 to 2017, the serum samples of 7,759 allergic patients collected from 38 hospitals in Yunnan were detected the specific immunoglobulin E (sIgE) against 8 indoor and food allergens, namely, house dust mite, cockroach, dog dander, mold mix, egg white, milk, crab, and shrimp. The polysensitization patterns were analyzed through cluster analysis, and the relationship between cockroach and other indoor and food allergens was analyzed.

Results: Allergen sIgE positivity was prevalent in $45.6 \%$ of the population. Cockroach was the most common allergen (27.0\%), followed by house dust mite (25.6\%), shrimp (18.8\%) and crab (15.6\%). Three polysensitization clusters were identified: cluster 1): egg white/milk; cluster 2): crab/shrimp/cockroach/house dust mite/dog dander; and cluster 3): mold mix. The sIgE levels and sensitization rates to house dust mite, crab, and shrimp increased with the level of cockroach sIgE $(P<0.05)$.

Conclusions: Based on big data in the real world, we found that there is a new trend in common allergens in Southwest China, where house dust mite is the only available reagent of specific immunotherapy. Cockroaches may become another major allergen in mainland China in the future, and clinicians should be aware of this.
\end{abstract}

Key words: cockroach allergen, polysensitization patterns, food allergen, allergic diseases, Specific IgE

\footnotetext{
From:

State Key Laboratory Respiratory Disease, National Clinical Research Center for Respiratory Disease, Guangzhou Institute of Respiratory Health, The First Affiliated Hospital of Guangzhou Medical University, Guangzhou Medical University, Guangzhou, China

${ }^{2}$ Longgang District People’s Hospital, Shenzhen, China

Contributed equally to this work.
}

\section{Introduction}

Despite the continuously increasing prevalence of allergic diseases, the causes remain unclear. It is well known that the interaction of genetic and environmental factors contributes to the increase in allergic disease, with exposure to environmental factors being a particularly notable factor in the development of allergic diseases. A meta-analysis in China showed that exposure to indoor allergens are the key risk factors for asthma. ${ }^{1}$ Indoor allergens, such as house dust mite, cockroach,

\author{
Corresponding author: \\ Baoqing Sun \\ 151 Yanjiang Road, Guangzhou 510120, China \\ E-mail: sunbaoqing@vip.163.com \\ Huimin Huang \\ 151 Yanjiang Road, Guangzhou 510120, China \\ E-mail: huanghuimin311@126.com
}

animal dander and mold, also play a major role in allergic diseases including asthma, allergic rhinitis, and skin allergy. ${ }^{2}$ Allergy patients are often sensitized to multiple allergens, including inhalants and food allergens (i.e., polysensitization). ${ }^{3}$ A follow-up study reported that $50 \%$ of children with positive test result for milk-IgE antibodies developed allergic rhinitis and asthma symptoms caused by inhalant allergens. ${ }^{4}$ Concurrently, patients with shellfish allergy (e.g., shrimp or crab) 
are often sensitized to indoor allergens such as house dust mite or cockroach. ${ }^{5}$ Therefore, a polysensitization profile is crucial for determining the existence of cross-reactivity to similar allergens or co-sensitization to allergens found in the same environment. ${ }^{6}$

The positive rate of cockroach allergen has also recently increased in the mountainous areas in southern China. ${ }^{8}$ Our previous studies found that the sensitization rate to cockroach allergen in inland areas was slightly higher than that in coastal areas (26.5\% vs. $20.9 \%)^{7}$ Therefore, regular, local-level epidemiological studies on allergens is important to improve evidence-based prevention of local allergens, particularly in large countries with a huge population like China.

Yunnan Province $\left(97^{\circ} 31^{\prime} \mathrm{E}-106^{\circ} 11^{\prime} \mathrm{E}, 21^{\circ} 09^{\prime} \mathrm{N}-29^{\circ} 15^{\prime} \mathrm{N}\right.$ ) is located in the inland of Southwest China and has a total population of 47.7 million as of 2016. Yunan Province is located in Yungui highland, and the area is characterized by high mountains and deep valleys, highly dense mountain streams, and lush vegetation; further, it has diverse climatic zones (tropical, temperate, and frigid). ${ }^{8}$

Although there have been several studies reporting on the prevalence of partial inhalant and food allergens in Yunnan Province, ${ }^{9,10}$ in-depth studies on polysensitization patterns and the relationship between indoor allergens, particularly cockroach, and food allergens, have been sparse both locally and globally.

Thus, this population-based cross-sectional observational study aimed to analyze the sIgE levels and sensitization rates of common indoor and food allergens in inland regions of Southwest China, as well as the polysensitization patterns of allergy patients, and investigate the relationship between cockroach and other allergens.

\section{Materials and methods \\ Study design and population}

This cross-sectional observational study reviewed the sIgE levels and sensitization rates to allergens in Yunnan Province from January 2016 to December 2017. Given the unavailability of modern laboratories for allergy diagnosis in Yunnan owing to economic constraints, the serum samples of allergy patients were delivered to a certified third-party laboratory service provider, the ImmunoCAP laboratory under KingMed Center for Clinical Laboratory Co. Ltd. (http://en.kingmed.com.cn/, which is an ISO-accredited [ISO/IEC17025, ISO9001, and ISO15189] and College of American Pathologists and American National Glycohemoglobin Standardization Program-recognized establishment) to ensure uniformity in allergen testing. During the 2 -year study period, majority of the serum samples were collected from 38 hospitals (13 secondary and 25 tertiary hospitals) in Yunnan.

We included patients who were tested for serum allergen sIgE and presented with clinical symptoms of suspected atopic diseases, with the following symptoms of allergies: allergic rhinitis (such as runny nose, sneezing, itching or nasal obstruction), symptoms of skin allergy (such as rashes, wheal, eczema, or urticaria), allergic asthma (such as wheezing, dyspnea, and/ or cough not due to common cold). The exclusion criterias were as below: samples from those parasitic infection, with immunodeficiency, or under specific immunotherapy. We recorded the doctor diagnoses, demographic data, and sIgE level for all allergy patients. The study flow chart was shown in Figure $\mathbf{1}$.

\section{Laboratory analysis \\ Sample collection, processing, and storage}

For each qualified patient, $5 \mathrm{ml}$ of venous blood was collected in separation gel-containing vacutainer tubes. The samples

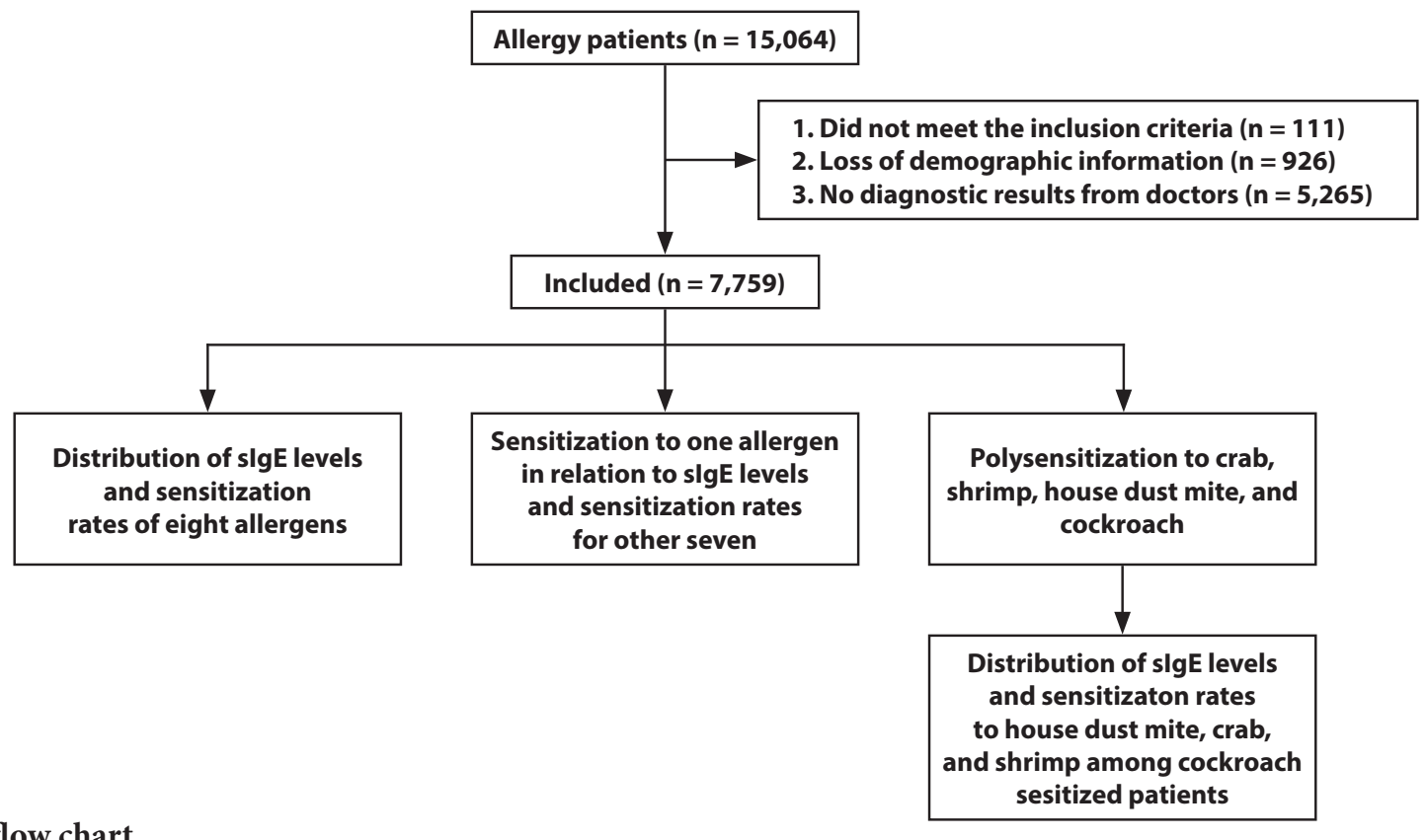

Figure 1. Study flow chart 
were then centrifuged at $3000 \mathrm{rpm}$ for 10 minutes, after which serum in the upper layer was collected and refrigerated at $-80^{\circ} \mathrm{C}$ for long term to avoid repeated freeze thaws. After the serum samples were all collected, they were transported in cold -chain vehicles to the accredited laboratory for testing.

\section{Allergen-specific IgE detection}

A fully automated in vitro allergen detector (ImmunoCAP 1000 system, Thermo Fisher Scientific Inc., California, USA) was used to detect allergen-specific sIgE in the sera. Enzyme-linked fluorescence assay was performed according to the manufacturer's instructions for sIgE detection. All laboratory technicians have successfully completed the training for ImmunoCAP application engineers. SIgE against eight allergen categories (species source) ${ }^{7,11}$ was evaluated in this study, namely, house dust mite (Dermatophagoides pteronyssinus), ${ }^{11}$ cockroach (Blatella germanica), ${ }^{11,12}$ mold mix (Penicillium chrysogenum, Cladosporium herbarum, Aspergillus fumigatus, and Alternaria alternata), dog dander, crab, shrimp, egg white, and milk.

Positive reactivity was defined as an $\operatorname{sIgE}$ level of $\geq 0.35$ $\mathrm{kU} / \mathrm{L}$ (Class 1 or above). According to the absolute sIgE levels, the reactivity was categorized quantitatively into six: Class 1, $\geq 0.35 \mathrm{kUA} / \mathrm{L}$ to $<0.70 \mathrm{kUA} / \mathrm{L}$; Class $2, \geq 0.70 \mathrm{kUA} / \mathrm{L}$ to $<3.50$ $\mathrm{kUA} / \mathrm{L}$; Class 3, $\geq 3.50 \mathrm{kUA} / \mathrm{L}$ to $<17.50 \mathrm{kUA} / \mathrm{L}$; Class $4, \geq 17.50$ $\mathrm{kUA} / \mathrm{L}$ to $<50.00 \mathrm{kUA} / \mathrm{L}$; Class $5, \geq 50.00 \mathrm{kUA} / \mathrm{L}$ to $<100.00$ $\mathrm{kUA} / \mathrm{L}$; and Class 6, $\geq 100.00 \mathrm{kUA} / \mathrm{L}$.?

\section{Ethical consideration}

This study was approved by the Institutional Review Board of First Affiliated Hospital of Guangzhou Medical University (Approval number: GYFYY-2017-18). This study was approved by the Institutional Review Board of First Affiliated Hospital of Guangzhou Medical University (Approval number: GYFYY -2017-18). All or part of the subjects and data involved in this research have not been published. All participants signed written informed consent. All methods, including the laboratory investigation and study implementation, were conducted in accordance with Committee on Publication Ethics (COPE) guidelines, Declaration of Helsinki, and China laws and legislation on the use of human biological samples for research purposes.

\section{Statistical analysis}

IBM SPSS Statistics for Windows Version 22.0 (IBM Corp, Armonk, USA) were used to processed and analyzed Data. Chi-square test or Fisher's exact probability method was applied to analyze the differences of sIgE positivity of different groups. Cluster analysis was used to measure the similarity in allergens sIgE levels and to classify them into different clusters. Kruskal-Wallis test was used to compare the multiple-group differences in allergen sIgE levels. Correlation analysis among the groups was performed by calculating the Spearman correlation coefficient $\left(r_{s}\right)$. Radar chart analysis was applied to compare different characteristics of multiple objects. Statistically significant were defined as P-values below 0.05 .

\section{Results}

Demographic characteristics of the study population

A total of 7,759 patients with a median age of 17 years (range 4-40 years) were included in this study; of them, 3,750 (48.3\%) were children aged < 14 years, 3,616 (46.6\%) were men, and $3,536(45.6 \%)$ were sensitized to at least one allergen (Table 1).

Table 1. Demographic characteristics of the study population

\begin{tabular}{|c|c|}
\hline Variable & Total $(\mathbf{N}=7759)$ \\
\hline Age $(y)$, median $\left(Q_{1}-Q_{3}\right)$ & $17(4-40)$ \\
\hline \multicolumn{2}{|l|}{ Age group (y), n (\%) } \\
\hline $0-6$ years & $2660(34.3)$ \\
\hline $7-14$ years & $1090(14)$ \\
\hline $15-35$ years & $1685(21.7)$ \\
\hline $36-60$ years & $1759(22.7)$ \\
\hline$\geq 61$ years & $565(7.3)$ \\
\hline \multicolumn{2}{|l|}{ Gender, n (\%) } \\
\hline Male & $3616(46.6)$ \\
\hline Female & $4143(53.4)$ \\
\hline \multicolumn{2}{|c|}{ The number of positive combined allergens, $n(\%)$} \\
\hline 0 & $4223(54.4)$ \\
\hline 1 & $1391(17.9)$ \\
\hline 2 & $677(8.7)$ \\
\hline 3 & $417(5.4)$ \\
\hline 4 & $732(9.4)$ \\
\hline 5 & $242(3.1)$ \\
\hline$\geq 6$ & $75(1.0)$ \\
\hline
\end{tabular}

\section{Distribution of sIgE levels and sensitization rates of indoor and food allergens}

The highest sensitization rate among indoor allergens was that to cockroach (27\%), followed by house dust mite $(25.6 \%)$, mold mix (3.9\%), and dog dander (3.3\%) (Figure 2A). Meanwhile, the highest sensitization rate among food allergens was that to shrimp (18.8\%), followed by crab (15.6\%), egg white (9.5\%), and milk (7.4\%) (Figure 2A). The median IgE levels of the eight allergens in the positive patients ranged from 0.56 $\mathrm{kU} / \mathrm{L}$ to $1.85 \mathrm{kU} / \mathrm{L}$.

With chi-square test analysis, the positive rate of house dust mite was significantly different among different age groups $\left(\chi^{2}\right.$ $=164.04, P<0.001, d f=4)$, as well as cockroach $\left(\chi^{2}=436.71\right.$, $P<0.001)$, mold mix $\left(\chi^{2}=41.65, P<0.001\right)$, dog dander $\left(\chi^{2}\right.$ $=61.14, P<0.001), \mathrm{crab}\left(\chi^{2}=187.48, P<0.001\right)$, shrimp $\left(\chi^{2}\right.$ $=255.02, P<0.001)$, egg white $\left(\chi^{2}=766.49, P<0.001\right)$, and milk $\left(\chi^{2}=837.68, P<0.001\right)$. In patients aged $0-6$ years (infant group), indoor allergen with the highest sensitization rates was house dust mite $(24.1 \%)$, while egg white had the highest sensitization rates $(22.3 \%)$ among food allergens. In all other age groups, indoor allergens with the highest sensitization rate 

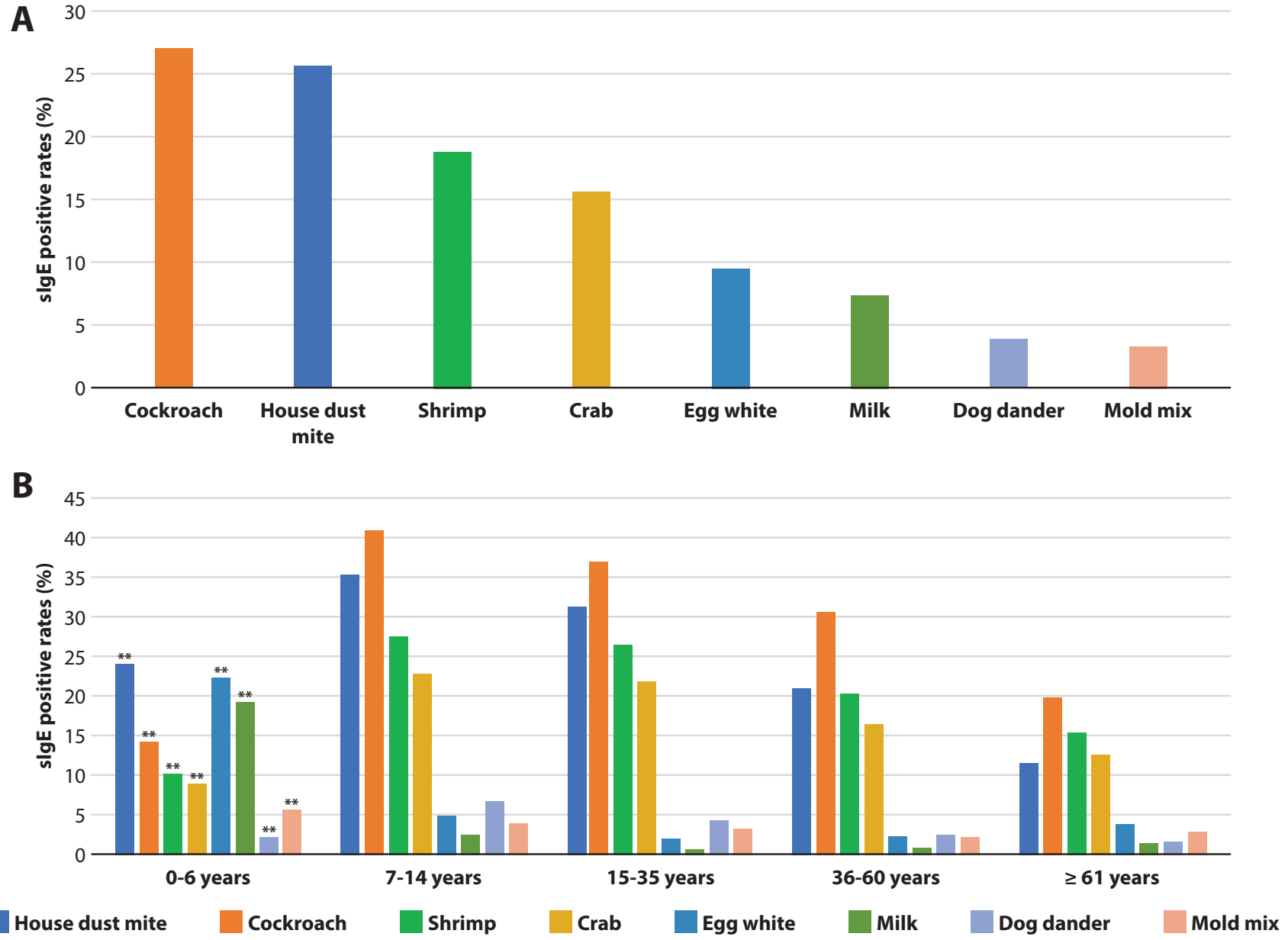

Figure 2. Distribution of positive sIgE rates against the studied allergens

(A) Overall allergen positive rate. (B) Allergen positive rate in different age groups.

$\star *$ With chi-square test analysis, the positive rate of house dust mite was significantly different among different age groups $\left(\chi^{2}=\right.$ 164.04, $P<0.001, d f=4)$, as well as cockroach $\left(\chi^{2}=436.71, P<0.001\right)$, mold mix $\left(\chi^{2}=41.65, P<0.001\right)$, dog dander $\left(\chi^{2}=61.14, P\right.$ $<0.001)$, crab $\left(\chi^{2}=187.48, P<0.001\right)$, shrimp $\left(\chi^{2}=255.02, P<0.001\right)$, egg white $\left(\chi^{2}=766.49, P<0.001\right)$, and milk $\left(\chi^{2}=837.68, P<\right.$ $0.001)$.

were cockroach (19.8\%-40.8\%), while food allergens with the highest sensitization rates were shrimp (15.4\%-27.5\%) (Figure 2B).

Sensitization to one allergen in relation to sIgE levels and sensitization rates for other seven allergens

In this study, sIgE against the eight allergens was evaluated, and we also explored the sIgE levels and sensitization rates to other seven allergens among patients sensitized to one allergen. As shown in the radar chart, patients sensitized to milk and egg, cockroach and house dust mite, crab and shrimp showed a similar sensitization trend to other seven allergens (Figure 3).

Among patients sensitized to crab, shrimp, and house dust mite, the most common cosensitization was to cockroach (96.4\%, 92.1\%, and 65.1\%), and the median sIgE levels were $1.96 \mathrm{kU} / \mathrm{L}, 1.69 \mathrm{kU} / \mathrm{L}$, and $0.79 \mathrm{kU} / \mathrm{L}$, respectively. Meanwhile, among cockroach-sensitized patients, the highest cosensitization was to shrimp (64.2\%), and the median IgE level was 0.54 $\mathrm{kU} / \mathrm{L}$. Among dog dander-sensitized and mold mix-sensitized patients, the highest cosensitization rate was to house dust mite (87.8\% and $62.1 \%$, respectively), and the median IgE levels were
$7.82 \mathrm{kU} / \mathrm{L}$ and $0.72 \mathrm{kU} / \mathrm{L}$, respectively. Among egg white-sensitized patients, the highest cosensitization rate was to milk $(40.8 \%)$, and the median IgE level of the positive patients was $0.88 \mathrm{kU} / \mathrm{L}$. Among milk-sensitized patients, the highest cosensitization rate was to egg whites $(52.9 \%)$, and the median $\operatorname{IgE}$ level of the positive patients was $1.00 \mathrm{kU} / \mathrm{L}$.

\section{Polysensitization patterns of allergy patients}

We analyzed the polysensitization patterns caused by the 8 allergens and classified them into the following three polysensitization clusters based on a cluster analysis on the similarity of sIgE levels (Figure 4): Cluster 1: egg white/milk; Cluster 2: house dust mite/cockroach/dog dander/crab/shrimp; Cluster 3: mold mix.

Strong correlations were observed among crab, shrimp, cockroach, house dust mite, and dog dander (all $r_{s} \geq 0.631$, all $P<0.05)$. A strong correlation was also found between milk and egg white $\left(r_{s}=0.609\right.$ and $\left.P<0.05\right)$. However, there was weak correlation between mold mix and other allergens (all $\mathrm{r}_{\mathrm{s}} \leq$ 0.385 , all $P<0.05$ ) (Figure 4). 
House dust mite

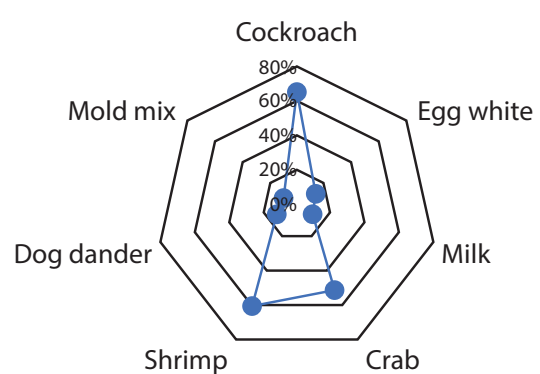

Crab

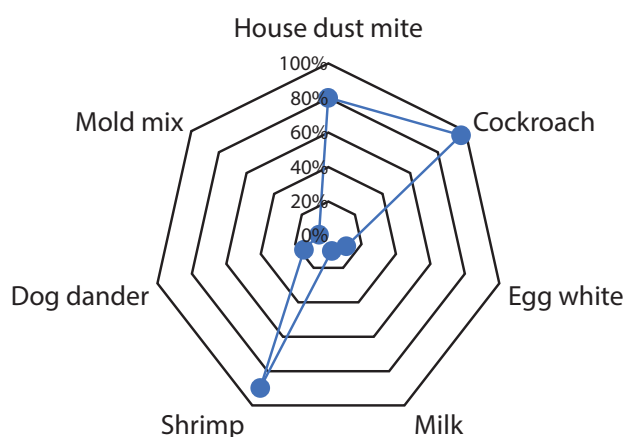

Egg white

House dust mite

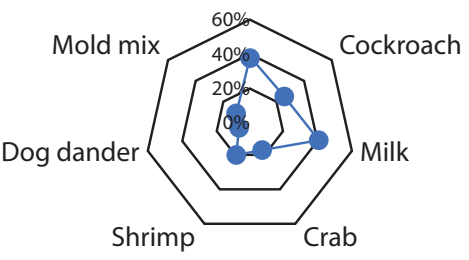

Dog dander

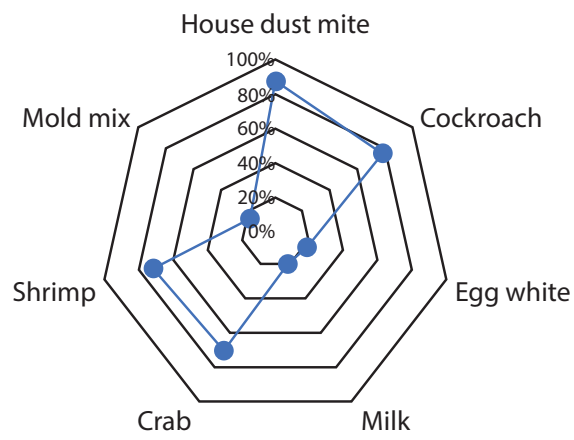

\section{Cockroach}

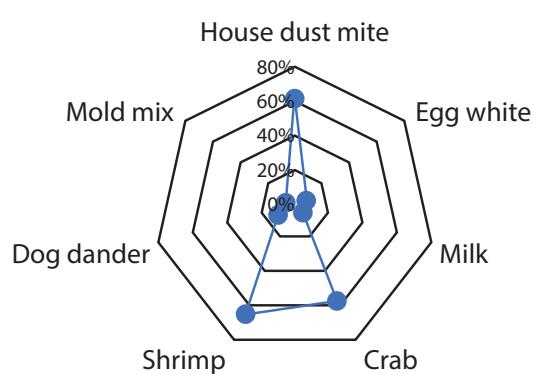

Shrimp

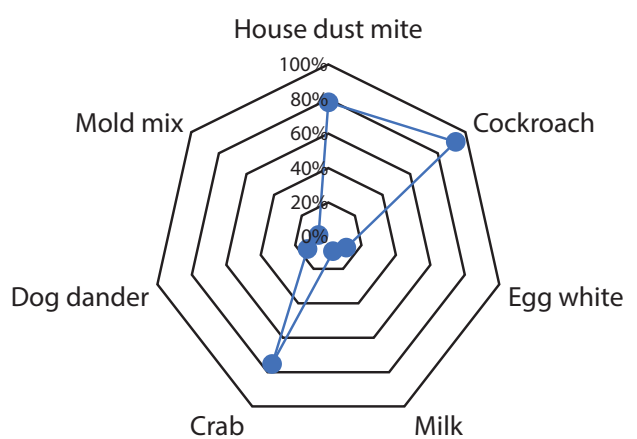

Milk

House dust mite

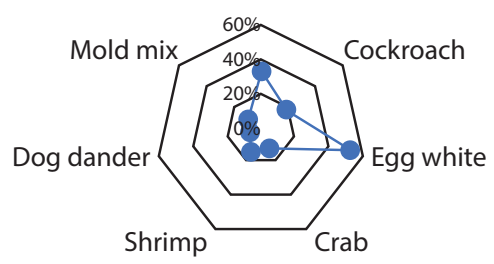

Mold mix

House dust mite

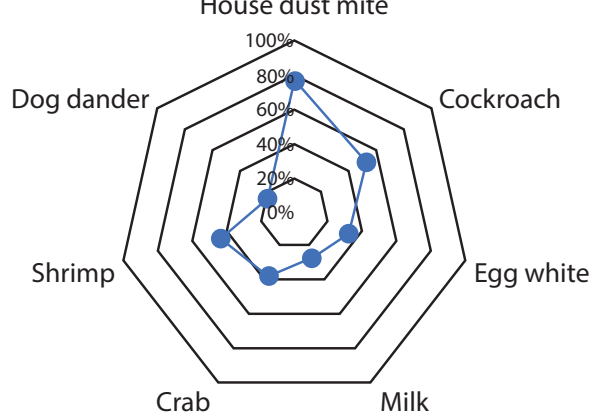

Figure 3. Radar chart of sensitization to one allergen in relation to sIgE levels and sensitization rates for other seven allergens Plots more closer to the outside of polygon processe that the allergen (at the top of the each subgraph) positive subjects were more likely to be sensitized for other allergens; plots more closer to the center processe that the allergen (at the top of the each subgraph) positive cases were less likely to be positive to other allergens. 


\begin{tabular}{|c|c|c|c|c|c|c|c|c|c|c|c|c|c|}
\hline 25 & 20 & 15 & 10 & 5 & & Crab & Shrimp & Cockroach & $\begin{array}{c}\text { House } \\
\text { dust mites }\end{array}$ & $\begin{array}{c}\text { Dog } \\
\text { dander }\end{array}$ & $\begin{array}{c}\text { Mold } \\
\text { mix }\end{array}$ & $\begin{array}{c}\text { Egg } \\
\text { white }\end{array}$ & Milk \\
\hline & & & & & Crab & 1 & & & & & & & \\
\hline & & & & & Shrimp & $0.851^{*}$ & 1 & & & & & & \\
\hline & & & & & Cockroach & $0.815^{*}$ & $0.827^{*}$ & 1 & & & & & \\
\hline & & & & & House dust mite & $0.721^{*}$ & $0.721^{*}$ & $0.698^{*}$ & 1 & & & & \\
\hline & & & & & Dog dander & $0.639^{*}$ & $0.638^{*}$ & $0.631^{*}$ & $0.646^{*}$ & 1 & & & \\
\hline & & & & & Mold mix & $0.248^{*}$ & $0.200^{*}$ & $0.241^{*}$ & $0.292^{*}$ & $0.385^{*}$ & 1 & & \\
\hline & & & & & Egg white & $0.229^{*}$ & $0.262^{*}$ & $0.217^{*}$ & $0.311^{*}$ & $0.376^{*}$ & $0.170^{*}$ & 1 & \\
\hline & & & & & Milk & $0.139 *$ & $0.184^{*}$ & $0.126^{*}$ & $0.219^{*}$ & $0.347^{*}$ & $0.141^{*}$ & $0.609^{*}$ & 1 \\
\hline
\end{tabular}

Cluster analysis

Spearman correlation coefficient $\left(r_{s}\right)$

Figure 4. Allergen cluster analysis and correlation analysis of allergy patients

*: Spearman correlation coefficient $\left(\mathrm{r}_{\mathrm{s}}\right), P<0.01$
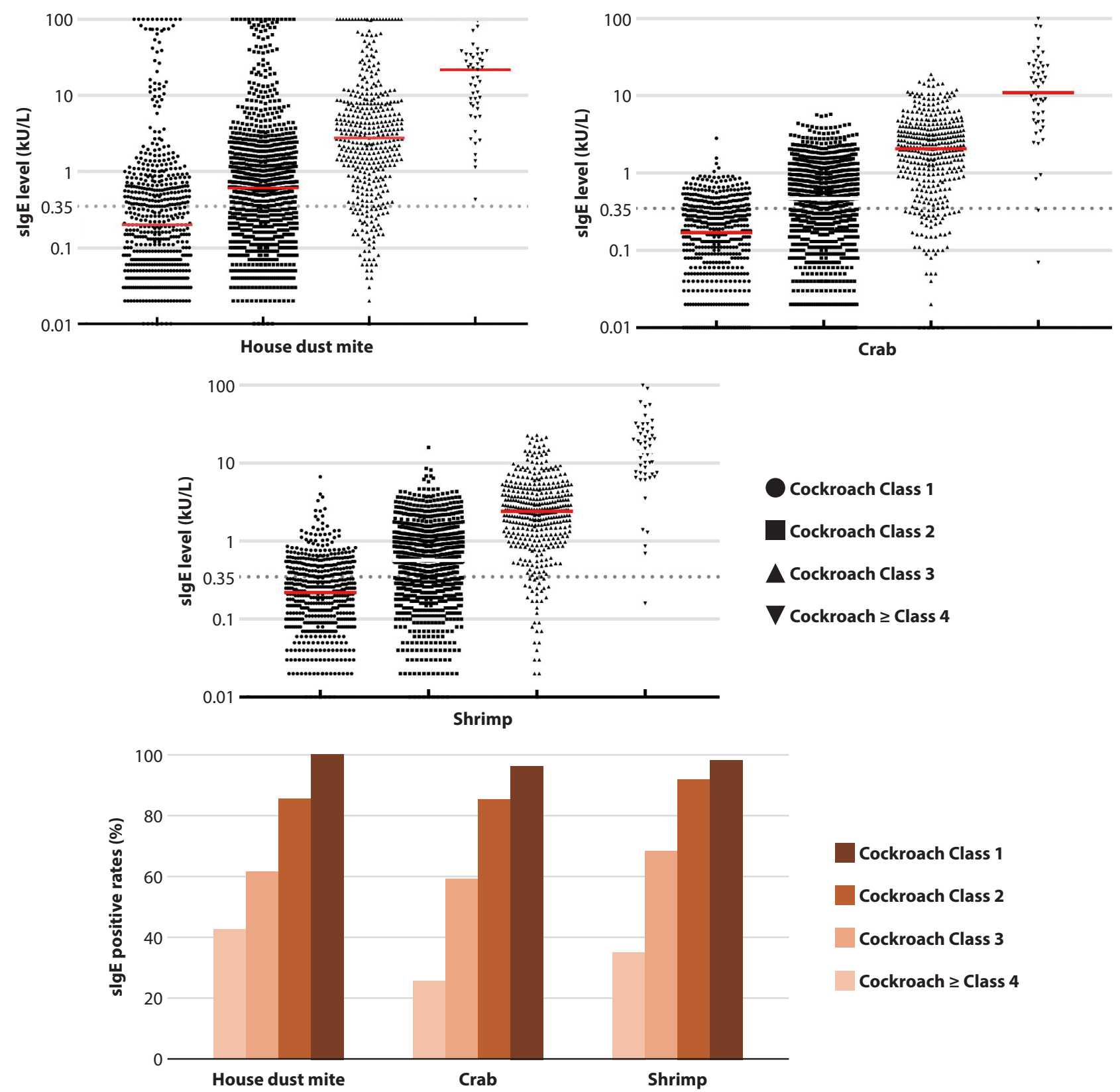

Cockroach Class 1

Cockroach Class 2

Cockroach Class 3

Cockroach $\geq$ Class 4

Figure 5. Distribution of sIgE levels and sensitization rates to house dust mite, crab, and shrimp among cockroach-sensitized patients 


\section{Distribution of Cluster 2-positive patients}

Among patients sensitized to Cluster 2 (house dust mite/ cockroach/dog dander/crab/shrimp) allergens, 37.0\% (2872/ 7759) were sensitized to at least one allergen, and the median sIgE levels against allergens in decreasing order were house dust mite (1.85 kU/L), cockroach (1.34 kU/L), crab (1.06 kU/L), shrimp $(0.96 \mathrm{kU} / \mathrm{L})$, and dog dander $(0.61 \mathrm{kU} / \mathrm{L})$.

Patients sensitized to at least one allergen were also often simultaneously sensitized to cockroach + house dust mite + crab + shrimp (26.9\%). House dust mite (20.3\%) and cockroach (16.4\%) were the top two allergens among mono-sensitized patients. Cockroach + house dust mite $(5.9 \%)$ was the most prevalent allergen among patients sensitized to two allergens. Cockroach + house dust mite + shrimp (4.9\%) accounted for the largest proportion of allergens among patients sensitized to three allergens. Meanwhile, only $5.7 \%$ of the population were sensitized to five allergens.

\section{Distribution of sIgE levels and sensitization rates of house dust mite, crab, and shrimp among cockroach-sensitized patients}

The relationship in sensitization between cockroach and other allergens (house dust mite, crab, and shrimp) were analyzed. We found that sIgE levels and sensitization rates of house dust mite, crab, and shrimp increased with the severity of cockroach-sIgE reactivity $(P<0.05)$.

When cockroach-sensitized patients showed low-class reactivity $(<0.70 \mathrm{kU} / \mathrm{L}$ or Class 1$)$, the median sIgE levels and sensitization rates of house dust mite, crab, and shrimp were usually mild (0.17-0.22 kU/L, 25.0\%-42.1\%). Meanwhile, when cockroach-sensitized patients showed high-class reactivity ( $\geq 0.70 \mathrm{kU} / \mathrm{L}$ or $\geq$ Class 2 ), the median sIgE levels and sensitization rates of house dust mite, crab, and shrimp significantly increased (0.47-21.8 kU/L, 59\%-100\%) and peaked at cockroach-sIgE class 4 and above reactivity (all $\geq 11 \mathrm{kU} / \mathrm{L}$, all $\geq 96 \%$ ) (Figure 5).

\section{Discussion}

Regional allergen epidemiological survey can provide evidence-based data for prevention, diagnosis, and treatment of local allergen. We investigated the sensitization to $8 \mathrm{com}-$ mon indoor and food allergens in the southwest inland region of China and found that the sensitization rates to cockroach (27.0\%) were slightly higher than that to house dust mite (25.6\%). A similar tendency was also found in a small, multicenter studies in China that investigated the allergen sIgE of eczematous dermatitis patients. ${ }^{13}$ In this study, the overall sensitization rates to cockroach were higher than that to house dust mite, with higher sensitization rate to cockroach primarily observed in adolescents and adults aged over 7 years. In our previous studies, we found that the positive rate of inhaled allergens increases with age and peaks 7-14 years. ${ }^{7,11}$ A similar finding was reported by Yang et al. who showed a higher prevalence of sensitization to cockroach in children aged 7-12 years living in the mountainous areas of southern China. ${ }^{14}$ This was similar to data from a large sample study of common allergen, in Japan, which showed that the positive rate of inhaled allergens peaked at age $10 .{ }^{15}$ Although this trend has not been reported by many epidemiological studies, the effects of exposure and sensitization to cockroach must not be overlooked: cockroach was found in 11\%-98\% of house dust samples collected from 9 cities across subtropical regions in southern China. ${ }^{12}$ De et al. reported that cockroach were detected in $85 \%$ of urban households in the US, and $60 \%-80 \%$ of children living in cities with asthma were sensitized to cockroach on skin prick test. ${ }^{16}$ For urban pediatric and adolescent asthma patients, asthma triggered by exposure and sensitization to cockroach even showed higher severity and more comorbidity than that caused by other indoor allergens such as dust mite or animal dander. ${ }^{17}$ According to the monthly measurements of indoor allergens conducted in households of northern cities in the US by Chew et al., the concentration of cockroach allergen Blag 1 peaked in June and August. Mollet et al. also reported that the level of cockroach allergen Blag 2 peaked in August, ${ }^{18}$ and this lasted for 2 months due to residuals and deposits of dead bodies, cast skins, and decrements. ${ }^{19,20}$

With respect to cases in which the sensitization rate to cockroach was slightly higher than that to dust mite detected in our study, we hypothesize that the level of cockroach allergen exposure may be corelated to the complex seasonal features of this inland region in recent years, but this needs to be confirmed by measuring the level of cockroach allergens in dust samples from local households. Several allergy patients are often sensitized to multiple allergies, ${ }^{21,22}$ which is probably caused by cross reactivity of allergens sharing similar epitopes or by co-exposure to allergens without similar epitopes. ${ }^{23.21}$ In this study, cluster analysis of allergen polysensitization among allergy patients allowed us to classify the cluster patterns for similar-origin or co-exposure allergens.

Jungyong et al. used the Multiple Allergen Simultaneous Test (MAST) to evaluate sIgE against 39 allergens among 4360 patients and classified these into 8 clusters as follows: cluster 1 : tomato, peach, rye, buckwheat, meal, citrus mix, rice, ragweed short, onion, barley meal, oak white, wheat flour, and birchalder mix; cluster 2: peanut, soybeans, mugwort, and cockroach; cluster 3: C. herbarum, A. alternata, A. fumigatus, and C. albicans; cluster 4: shrimp and crab; cluster 5: D. pteronyssinus, D. farinae, A. siro, house dust, dog, and cat; cluster 6: salmon, codfish, tuna, and baker's yeast; cluster 7: cheddar, beef, milk, and pork; and cluster 8: garlic, egg white, and chicken. ${ }^{25}$ Our study findings are partly similar with their findings, but there are also some differences: first, milk and egg were grouped in the same cluster, and among milk-sensitized or egg-sensitized patients, the sensitization rate to egg or milk were markedly higher than that to other allergens $(53.9 \%$ and $40.5 \%$, respectively). In a 5 -year study of 100 children with milk allergy, $58 \%$ of the children had an adverse reaction after eating eggs. ${ }^{26}$ It was also reported that children with milk allergy are more likely to be sensitized to egg by up to $41 \%$ to $67 \% .^{27,28}$ Second, shrimp, crab, cockroach, house dust mite, and dog dander were clustered together. Among dog dander-sensitized patients, the sensitization rates to house dust mite $(6.28$ $\mathrm{kU} / \mathrm{L})$ and cockroach $(4.21 \mathrm{kU} / \mathrm{L})$ reached a high level of at least $80 \%$, meaning that they may be the common triggers in the house dust..$^{29}$ Patients (26.9\%) cosensitized to shrimp, crab, cockroach, and house dust mite were most common in the cluster 2 . 
According to the clusters identified in this study, we recommend that patients confirmed to be sensitized to a particular allergen should be actively evaluated to sensitization to similar allergens in the tree diagram from the cluster analysis, and they should be informed about the other possible allergens in the same cluster.

Tropomyosin is the major allergen causing cross-reaction between shellfish. Tropomyosin of shrimps, lobsters, and crabs share sequence identity between $91 \%$ and $100 \%$. In our study, crab- or shrimp-sensitized patients showed high sensitization rates $\left(74.6 \%\right.$ and $90.1 \%$, respectively. ${ }^{30}$ Rosenfield et al. demonstrated that $90.5 \%$ of patients with shrimp allergy are sensitized to house dust mite on skin prick test. ${ }^{31}$ Similar results were also obtained in our study. Among crab-sensitized and shrimp-sensitized patients, sIgE levels and sensitization rates to house dust mite were both high $(1.42 \mathrm{kU} / \mathrm{L}$ and $1.13 \mathrm{kU} / \mathrm{L}$ [81.4\% and $79.2 \%$, respectively]). We also observed that up to more than $90 \%$ of patients sensitized to crab and shrimp were allergic to cockroach, with elevated IgE levels $(1.96 \mathrm{kU} / \mathrm{L}$ and $1.69 \mathrm{kU} / \mathrm{L}$ respectively). Yang et al discovered that in mildly symptomatic shrimp-sensitized children living in rural areas of southern China, cockroach is a major source of cross-reactive allergen source. ${ }^{32}$ Meanwhile, another study revealed that for children sensitized to cockroach and shrimp, exposure to high levels of cockroach allergen can contribute to higher IgE levels against shrimp, which might not correlate with clinical reactivity. ${ }^{33}$ In our study, only a small percentage of patients were mono-sensitized to crab $(0.5 \%)$ or shrimp (1.5\%). Meanwhile, sIgE levels and sensitization rates to house dust mite, crab, and shrimp increased with the severity of cockroach-sIgE reactivity $(P<0.05)$, and even increased at a rate higher than 95\% (all $\geq 11 \mathrm{kU} / \mathrm{L}$ ). Collectively, these results suggest that cross-reactivity or co-sensitization, particularly cross-reactivity to cockroach, should be considered in patients sensitized to cockroach, shrimp, crab, or house dust mite.

This study has certain limitations that should be considered when interpreting the findings. First, because it is a retrospective cross-sectional study, results on the sensitization feature of allergens only correspond to data of a particular time period. Further, considering the large number of patients in our study, a sampling bias could have occurred because the subjects included were identified via a preliminary diagnosis only based on the patient's medical history, allergic symptoms, and IgE levels, without the support of a complete clinical evaluation. Some of the observed results lack robustness to be applied in analyzing specific allergic diseases because of the lack of detailed clinical data. Only one kind of house dust mite allergen, D. pteronyssinus, was included in this study, while $D$. farinae or even $B$. tropicalis were not included for analysis, which was also a limitation of this study. Moreover, we were unable to study allergen components of cross-sensitization or co-sensitization among cockroach, house dust mite, shrimp, and crab due to the lack of available commercial reagents and conditions for detection.

\section{Conclusions}

In summary, we found a new trend in allergen sensitization in that cockroach was slightly more common than house dust mite among subjects with atopy, indicating that cockroach should be given importance in the management of allergen in this inland region. The common indoor and food allergens could be classified into three polysensitization patterns: egg white/milk, crab/shrimp/cockroach/house dust mite/dog dander, and mold mix. Cross-reactivity or co-sensitization, particularly cross-reactivity to cockroach, should be considered in patients sensitized to shrimp, crab, or house dust mite.

\section{Acknowledgements}

The authors thank Xiaoying Huang for their assistance in the data collection.

\section{Conflict of interests}

None declared.

\section{Funding sources for the study}

The authors thank the National Natural Science Foundation of China (81871736 and 81802076), National Key Technology R\&D Program (2018YFC1311900), Guangdong Science and Technology Foundation (2019B030316028), Guangzhou Municipal Health Foundation (20191A011073), SKLDR (MS201906), and Guangzhou Science and Technology Foundation (201804020043).

\section{Author contributions}

BS and WL conceived the study, designed the methods and coordinated data collection. WL and $\mathrm{HC}$ wrote the first draft of the paper. ZW, HC and HH contributed to data analysis. All authors critically revised the manuscript and approved the final version.

\section{References}

1. Yan C, Wong G W, Jing L. Environmental Exposure and Genetic Predisposition as Risk Factors for Asthma in China. Allergy Asthma Immunol Res. 2016; 8:92-100.

2. Tham EH, Lee AJ, Bever HV. Aeroallergen sensitization and allergic disease phenotypes in Asia. Asian Pac J Allergy Immunol. 2016;34:181-9.

3. de Jong AB, Dikkeschei LD, Brand PLP. Sensitization patterns to food and inhalant allergens in childhood: A comparison of non-sensitized, monosensitized, and polysensitized children. Pediatr Allergy Immunol. 2015;22:166-71.

4. Host A, Jacobsen HP, Halken S, Holmenlund D. The natural course of cows milk protein allergy/intolerance. European journal of clinical nutrition. 1995;49:S13-8.

5. Lopata AL, Lehrer SB. New insights into seafood allergy. Curr Opin Allergy Clin Immunol. 2009;9:270-7.

6. Roberts G, Peckitt C, Northstone K, Strachan D, Lack G, Henderson J, Golding J. Relationship between aeroallergen and food allergen sensitization in childhood. Clin Exp Allergy. 2005;35:933-40.

7. Zeng GQ, Luo WT, Wu ZH, Li L, Zheng PY, Huang HM, et al. A cross -sectional observational study on allergen-specific IgE positivity in a southeast coastal versus a southwest inland region of China. Sci Rep. 2017; 7:9593.

8. yn.gov.cn [Internet]. China: People's government of yunnan province; c2019 [cited 2019 Oct 28]. Available from: http://www.yn.gov.cn/yngk/gk/ 201904/t20190403_96251.html

9. Li JP. Epidemiology survey and risk factors of bronchial asthma in Kunming. Clin Pulmonary Medicine. 2015;20:1667-9. Chinese. 
10. Li SS, Yu YM, Ruan B, Liu B, Zhao YY, Qiu JW. Prevalence of sensitization to aeroallergens in 1893 patients with allergic rhinitis in Yunnan. Clinical Otorhinolaryngology Head and Neck Surgery. 2013;27:246-50. Chinese.

11. Sun BQ, Chen DH, Zheng PY, Huang HM, Luo WT, Zeng GQ. Allergy-related evidences in relation to serum IgE: Data from the China State Key Laboratory of Respiratory Disease, 2008-2013. Biomed Environ Sci. 2014;27:495-505.

12. Zheng YW, Lai XX, Zhao Y, Zhang CQ, Chen JJ, Zhang L, et al. Indoor Allergen Levels and Household Distributions in Nine Cities Across China. Biomed Environ Sci. 2015;28:709-17.

13. Lu XY, Wang DX, Jiang Jg, Li QF, Zhou X, Chen L, et al. Detection of allergen-specific serum IgE in patients with eczema or dermatitis: a multicenter study. Chinese Journal of Dermatology. 2015(9):616-20. Chinese.

14. Yang Z, Zheng W, Yung E, Zhong N, Wong GWK, Li J. Frequency of food group consumption and risk of allergic disease and sensitization in schoolchildren in urban and rural China. Clin Exp Allergy. 2015;45: 1823-32.

15. Kimura S, Chen GL. A large-scale statistical analysis of positive ratios of allergen specific IgE antibodies in serum. J Diagn Concepts Pract. 2002;1: 40-1.

16. Do DC, Zhao Y, Gao P. Cockroach allergen exposure and risk of asthma. Allergy. 2016;71:463-74.

17. Rosenstreich DL, Eggleston P, Kattan M, Baker D, Slavin RG, Gergen P, Mitchell $\mathrm{H}$, et al. The role of cockroach allergy and exposure to cockroach allergen in causing morbidity among inner-city children with asthma. N Engl J Med. 1997;336:1356-63.

18. Chew GL, Higgins KM, Gold DR, Muilenberg ML, Burge HA. Monthly measurements of indoor allergens and the influence of housing type in a northeastern US city. Allergy. 2015;54:1058-66.

19. Mollet JA, Vailes LD, Avner DB, Perzanowski MS, Arruda LK, Chapman $\mathrm{MD}$, et al. Evaluation of German cockroach (Orthoptera:Blattellidae) allergen and seasonal variation in low-income housing. Medical Entomology. 1997;34:307-11.

20. Lehrer SB, Horner WE, Menon P, Stankus RP. Comparison of cockroach allergenic activity in whole body and fecal extracts. J Allergy Clin Immunol. 1991;87:574-80.
21. Giorgio C, Cristoforo I, Paola P, Soffia S, Scurati S, Frati F. Polysensitization as a challenge for the allergist:the suggestions provided by the Polysensitization Impact on Allergen Immunotherapy studies. Expert Opinion on Biological Therapy. 2011;11:715-22.

22. Prigione I, Morandi F, Tosca MA, Silvestri M, Pistoia V, Ciprandi G, et al. Interferon-gamma and IL-10 may protect from allergic polysensitization in children:preliminary evidence. Allergy. 2010;65:740-2.

23. Scala E, Alessandri C, Bernardi ML, Ferrara R, Palazzo P, Pomponi D, et al. Cross-sectional survey on immunoglobulin E reactivity in 23077 subjects using an allergenic molecule-based microarray detection system. Clin Exp Allergy. 2010;40:911-21.

24. Soeria-Atmadja D, Onell A, Kober A, Matsson P, Gustafsson MG, Hammerling U. Multivariate statistical analysis of large-scale IgE antibody measurements reveals allergen extract relationships in sensitized individuals. J Allergy Clin Immunol. 2007;120:1433-40.

25. Ohn J, Paik SH, Doh EJ, Park HS, Yoon HS, Cho S. Allergen Sensitization Pattern by Sex: A Cluster Analysis in Korea. Ann Dermatology. 2017;29: 735-41.

26. Bishop JM, Hill DJ, Hosking CS. Natural history of cow milk allergy: clinical outcome. J Pediatr. 1990;116:862-7.

27. Host A, Jacobsen HP, Halken S, Holmenlund D. The natural history of cow's milk protein allergy/intolerance. Eur J Clin Nutr. 1995;49:S13-8.

28. Hill DJ, Bannister DG, Hosking CS, Kemp AS. Cow milk allergy within the spectrum of atopic disorders. Clin Exp Allergy. 1994:24:1137-43.

29. Stemeseder T, Schweidler B, Doppler P, Klinglmayr E, Moser S, Lueftenegger L, et al. Exposure to Indoor Allergens in Different Residential Settings and Its Influence on IgE Sensitization in a Geographically Confined Austrian Cohort. PLoS One. 2017;12:e0168686.

30. Wong L, Huang CH, Lee BW. Shellfish and house dust mite allergies: is the link tropomyosin? Allergy Asthma Immunol Res. 2016;8:101-6.

31. Rosenfield L, Tsoulis MW, Milio K, Schnittke M, Kim H. High rate of house dust mite sensitization in a shrimp allergic southern Ontario population. Allergy Asthma Clin Immunol. 2017;13:5.

32. Yang Z, Zhao J, Wei N, Schnittke M, Kim H. Cockroach is a major cross-reactive allergen source in shrimp-sensitized rural children in southern China. Allergy. 2017;73.

33. Wang J, Calatroni A, Visness CM, Sampson HA. Correlation of specific IgE to shrimp with cockroach and dust mite exposure and sensitization in an inner-city population. J Allergy Clin Immunol. 2011;128:834-7. 